\title{
Association of osteoarthritis and circulating adiponectin levels: a systematic review and meta-analysis
}

\author{
Qian Tang ${ }^{1 \dagger}$, Zhi-Chao Hu${ }^{1 \dagger}$, Li-Yan Shen ${ }^{1}$, Ping Shang ${ }^{2}$, Hua-Zi Xu ${ }^{1 *}$ and Hai-Xiao Liu ${ }^{1 *}$ (D)
}

\begin{abstract}
Background: The objective of this study was to perform a meta-analysis to investigate the specific relationship between the expression level of circulating adiponectin and osteoarthritis (OA).

Method: Multiple databases were searched to estimate the high quality of studies relevant to adiponectin and OA. We extracted the data from the eligible studies and included them in the meta-analysis using a random effects model. Subgroup analysis and meta-regression were further performed to explore the potential sources of heterogeneity.

Results: Ten articles consisting of thirteen case-control studies that contained a combined total of 1255 subjects. Our results revealed that the $\mathrm{OA}$ patients displayed higher adiponectin levels than the healthy controls (SMD $=0.327$, 95\% Cl: $0.11-0.55, P=0.003)$. The ethnicity-stratified subgroup analysis indicated that the adiponectin was a sensitive biomarker in both Caucasians ( $P=0.021)$ and Asians $(P=0.037)$. Moreover, the meta-regression analysis suggested that the sample size $(P=0.03)$ and nationality $(p=0.01)$ could account for a part of heterogeneity in our study.

Conclusion: Taken together, the current study indicated that the adiponectin expression levels were higher in the OA patients than in the healthy controls and might be associated with OA prevalence.
\end{abstract}

Keywords: Osteoarthritis, Adiponectin, Meta-analysis

\section{Background}

Osteoarthritis (OA), a painful degenerative joint disease involving articular cartilage loss, subchondral bone remodeling, osteophyte formation and soft tissue damage, causes ongoing disability in elderly people due to its irreversible outcome $[1,2]$. The worldwide incidences of knee and hip OA are estimated to be $6-17 \%$ and $2-10 \%$, respectively and the condition especially affects women over 60 years of age [3]. It has become the main reason for total joint arthroplasty (TJA) and is therefore to the source of a considerable economic burden [4]. While the etiology of osteoarthritis $[\mathrm{OA}]$ is still not clearly understood $[5,6]$, the evidence suggested that $\mathrm{OA}$ is a systemic disorder with a multifactorial origin. The systemic risk factors include obesity, gender, injury, age and a genetic bias

\footnotetext{
* Correspondence: spine-xu@163.com; spineliu@163.com

${ }^{+}$Qian Tang and Zhichao Hu contributed equally to this work.

'Department of Orthopaedic Surgery, The Second Affiliated Hospital and

Yuying Children's Hospital of Wenzhou Medical University, 109, Xueyuanxi

road, Wenzhou 325027, China

Full list of author information is available at the end of the article
}

[2]. Moreover, there are increasing evidences indicated that obesity is an essential element in the pathogenesis of OA [7]. Sowers et al. [8] have suggested that the mechanism by which obesity is involved in OA, may be a simple increase under the mechanical burden in the joints. However, Gabay et al. [9] indicated that the obesity induced high metabolic and inflammatory environments play crucial roles in the onset of OA. What's more, several early studies have indicated that there is an association between $\mathrm{OA}$ and some adipokines in serum or synovial fluid [10-13]. One of these mediators of interest is adiponectin, which has been shown to be in relation to OA [14].

Adiponectin, a $28-30 \mathrm{kDa}$ collagen-like protein, not only is one of the most abundantly secreted adipose tissue proteins but also is the only adipokine identified thus far that is negatively correlated with obesity [15]. For many years, the effects of adiponectin in many metabolic conditions such as insulin resistance, atherosclerosis, and myocardial infarction have been intensely studied [16-18], 
as well as its roles in inflammatory and anti-inflammatory processes [19]. However, the effects of adiponectin in OA process were still controversial. On the one hand, evidence had shown that both human and murine chondrocytes express functional adiponectin receptors (AdipoR1 and AdipoR2) [20]. In cultured chondrocytes, adiponectin treatment leads to a dose-dependent increase of the pro-inflammatory factors such as inducible nitric oxide synthase (iNOS), interleukin-6 (IL-6) and metalloproteases (MMPs) [21, 22]. All of those factors may degrade matrix, destroy articular cartilage and eventually result in OA [23]. What's more, several studies supported that the higher level of adiponectin was presented in both the plasma and synovial fluid of OA patients compared with the healthy controls [14, 24-29]. In addition, a recent study found there was a positive correlation between adiponectin concentration and the Kellgren-Lawrence (KL) grading scores (A scoring system describes the degree of joint degeneration via X-ray image system) [14]. On the other hand, a few studies have failed to demonstrate a statistical association between adiponectin and OA [20, 30,31]. Moreover, some data suggest that the adiponectin expression level is negatively correlated with the radiographic severity of $\mathrm{OA}$ and might be playing a protective role in the pathogenesis of OA [32, 33]. To examine what role the adiponectin plays in OA process, we conducted this meta-analysis to evaluate the relationship between the expression level of adiponectin and OA prevalence.

\section{Methods}

\section{Searching strategy}

In this meta-analysis, we followed the guidelines regarding the preferred reporting items recommended in the systematic reviews and meta-analyses (PRISMA) statement [34] to identify the relevant studies written in English or non-English languages, which were found by searching the following electronic databases: MEDLINE, Embase, Cochrane Library, China National Knowledge Infrastructure (CNKI), and Google Scholar. In addition, further relevant studies including those shown in the reference lists of all the included studies were also manually searched. The last search was carried out on August 10, 2017. The following search terms ("Osteoarthritis, Knee" or "Osteoarthritis, hip" or "Osteoarthritis, spine" or "Osteoarthritis" or "knee osteoarthritis" or "hip osteoarthritis" or "spine osteoarthritis" or "spinal osteoarthritis" or "lumbar osteoarthritis" or "hand osteoarthritis") and ("Adiponectin" or "adiponectin" or "Obese Protein" or "Obese Gene Product") were used. The search strategy is presented in Fig. 1.

\section{Selection criteria}

Studies meeting the following criteria were included: 1) the basic requirement was that the studies were restricted to human populations and published in a peer-reviewed journal; 2) only case-control or cohort studies that measured the expression levels of adiponectin in both osteoarthritis patients and healthy controls were included; 3 ) a minimum sample size of least ten was required; 4) all of the OA patients were identified and confirmed by the Diagnostic and Therapeutic Criteria Committee of the American Rheumatism Association [35]; 5) the original data and sufficient information were provided regarding adiponectin serum or synovial levels in the OA patients and healthy groups. The major exclusion criteria were as follows: 1 ) the studies did not satisfy the above requirements; 2) the studies included duplicate publications, for example, studies published by the same author or having data derived from the same clinical trial.

\section{Data extraction}

For each eligible study, two of the authors of the present study independently extracted all of the relevant data following the criteria above, and disagreements were resolved by discussion with a third investigator. For each publication, the following variables were abstracted: the surname of the first author, publication year, source of publication, study design, sample size, patient genders, mean age, Body Mass Index (BMI), country, ethnicity, joint involvement, stage of OA, expression level of adiponectin, source of the samples from the controls and subjects used for the adiponectin analysis and the detection method. To evaluate the effects of the various ethnicities of the subjects, we collected this information separately and classified the subjects into Asians and Caucasians. And the subgroup evaluation was also conducted by dividing parts into two groups according to the KL score or radiological findings: early OA group (KL score $=1$ or without radiological change) and middle and late OA group (KL score $=2,3,4$ or with radiological change). All authors approved the final inclusion of studies for meta-analysis.

\section{Quality assessment}

To ensure a high quality assessment of the included studies, two experienced reviewers independently applied the Newcastle-Ottawa scale (NOS) [36] Using this tool, each study was judged on eight items, categorized into the following three groups: 1) the selection of the study groups; 2 ) the comparability of the groups; 3 ) the assessment of either the exposure or the outcome of interest for case-control or cohort studies, respectively. Stars were awarded for each quality item to serve as a quick visual assessment. Up to nine stars were awarded to the highest quality studies. A third reviewer was consulted if there had been any disagreement between the two investigators regarding the any of the elements of NOS scores. 


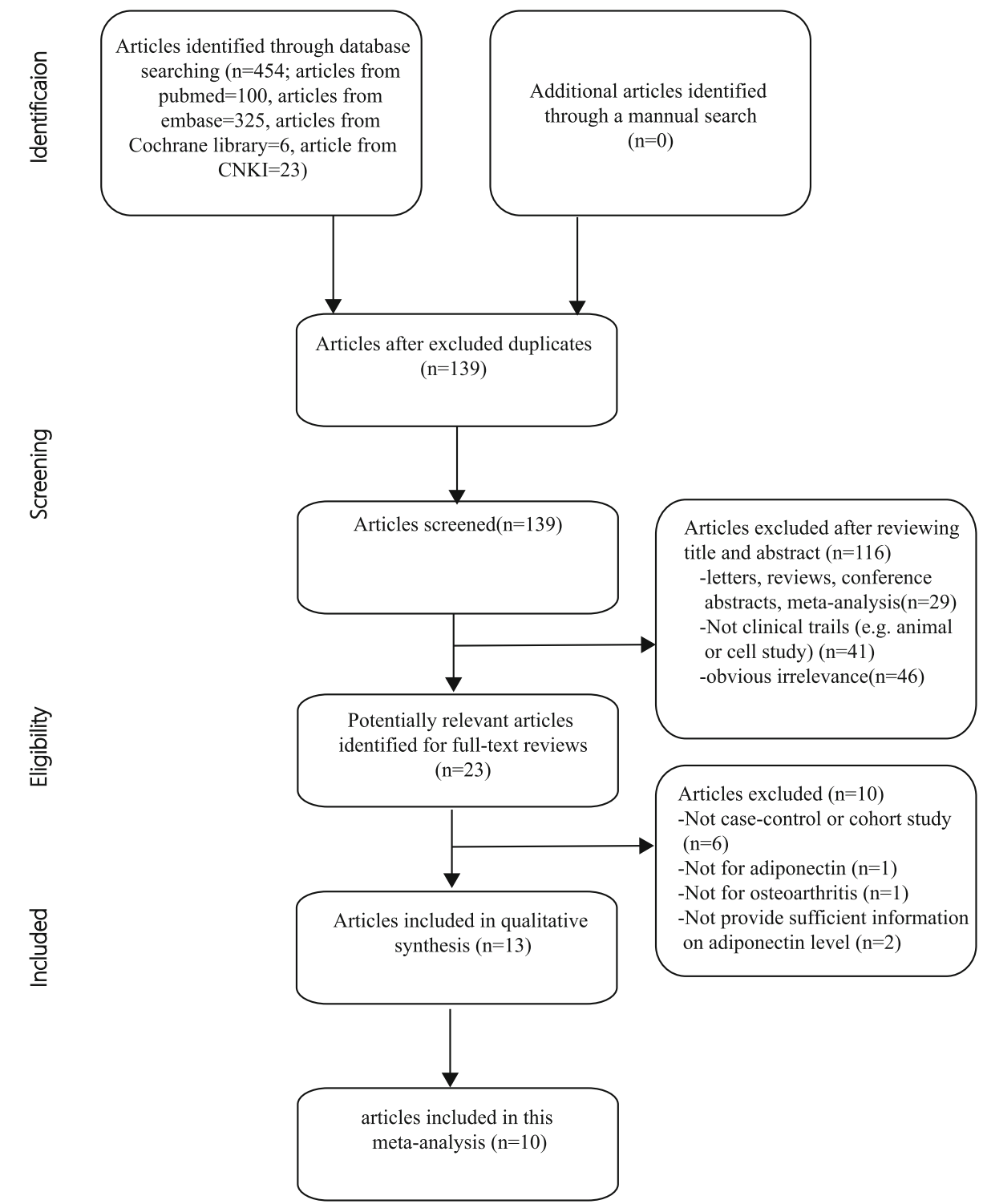

Fig. 1 Flow chart of the articles selection and inclusion process

\section{Statistical analysis}

This meta-analysis was undertaken using STATA 12.0 software (Stata Corp, College Station, TX, USA). A standardized mean difference (SMD) for the adiponectin expression levels and the 95\% CIs were calculated for the generic inverse variance outcomes between the cases and controls in accordance with the Z-test. According to the statistical heterogeneity was considered to be present at $P<0.05$ and the $\mathrm{I}^{2}$ values were $>50 \%$ in our study, a random effects model was applied and further subgroup study and meta-regression analysis were performed to detect the origin of heterogeneity. To test the strength and stability of the pooled results, we performed a sensitivity analysis by omitting the individual studies one by one.
Moreover, the effect of publication bias was investigated by egger's test.

\section{Results}

\section{Literature search outcome}

The search strategy (Fig. 1) identified 454 relevant studies from the databases listed above. After reviewing the titles, abstracts and the full-text of the articles, only ten articles consisting of thirteen case-control studies, which included 754 OA patients and 501 healthy controls, remained for our meta-analysis. The quality scores for the methods used in the thirteen included studies are presented in the end of Table 1. Information on the joint involvement, subjects' ethnicity, the stage of OA and source of samples 


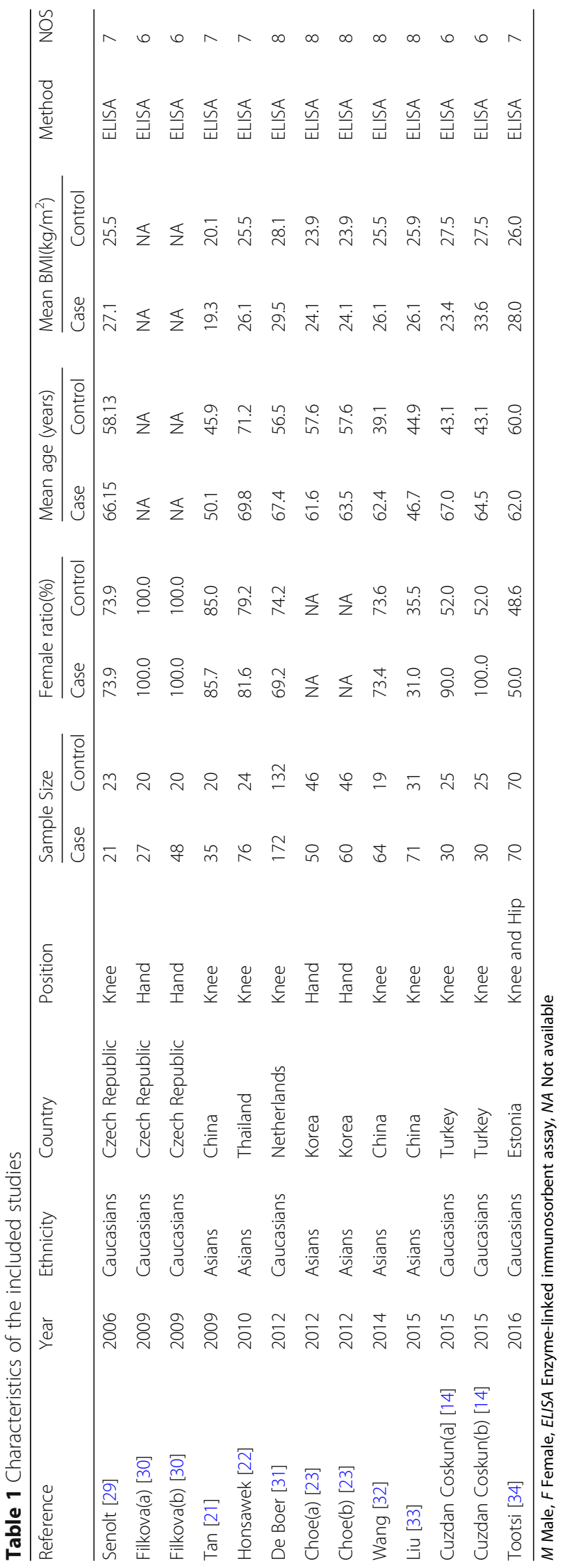


was provided in all of these studies. The BMI scales were provided in eleven studies. Table 1 summarizes the basic characteristics and the information provided in the included studies. The scores for the quality of the methodology of the ten included studies are also presented in Table 1.

\section{Study characteristics}

The pooled results indicated that the expression level of adiponectin was significantly higher in the OA patients compared with that in the healthy groups $(\mathrm{SMD}=0.327$, 95\% CI: $0.11-0.55, P=0.003$, Fig. 2). The causes of heterogeneity in the results were explored according to our a priori hypotheses by subgroup analysis and meta-regression. The results of subgroup analysis showed that the higher expression level of adiponectin was observed only in the knee OA patients, but was not significantly observed in the subjects with hand OA or knee and hip OA. (Knee OA: SMD $=0.44,95 \%$ CI: $0.17-0.72, P=0.002$; Hand $\mathrm{OA}: \mathrm{SMD}=0.18,95 \% \mathrm{CI}$ : $-0.07-0.44, P=0.161$; Knee and hip OA: $\mathrm{SMD}=0.00$, 95\% CI: $-0.33-0.34, P=0.980$, Table 2). Meanwhile, the results indicated that adiponectin might be a biomarker for OA in both Caucasian and Asian subjects as analyzed in current study (Caucasians: SMD $=0.42,95 \% \mathrm{CI}$ : $0.06-0.71, P=0.021$; Asians: SMD $=0.19,95 \%$ CI: $0.01-$ $0.18, P=0.037$, Table 2). What's more, the stage-stratified analysis showed that the adiponectin expression levels were correlated with $\mathrm{OA}$ in the patient who entered the later stage of OA, but in the early OA patients group, we failed to get the similar results (early OA: $\mathrm{SMD}=0.00$,
95\% CI: $-0.57-0.58, P=0.356$; middle and late OA: $\mathrm{SMD}=$ 0.23 , 95\% CI: $-0.17-0.63, P=0.004$, Table 2). In addition, the separate variable and multivariable meta-regression analyses for adiponectin expression level were performed to analyze the potential sources of inter-study heterogeneity. Overall, the sample size and the nationality of patients might be the major sources of heterogeneity for our study (Sample size: $p=0.03$; Korea vs Czech Republic: $p=0.01$, Table 3). Furthermore, the sensitivity analysis indicated that no significant differences resulted from the omission of the data from any single study (Fig. 3). and the Egger's test showed that there is no publication bias in this metaanalysis ( $t=-1.58, P=0.144$, Fig. 4).

\section{Discussion}

In this meta-analysis, the major finding was that expression level of adiponectin was significantly higher in the OA patients than in healthy subjects. This result might support those of a previous study that indicated that the metabolism of adipose tissue was a key factor that contributed to the OA development [37] and the adiponectin might be closely related to the pathogenesis of OA.

However, with respect to the molecular mechanism, it is still a matter of dispute whether adiponectin plays a protective role or an effector role. Some investigators considered its function to be primarily a pro-inflammatory mediator that may cause OA [10], whereas others hold the opposite opinion [20]. In clinical studies, the relationship between the adiponectin levels and the severity of the $\mathrm{OA}$ is also controversial. Most investigators have suggested that adiponectin plays a protective role in

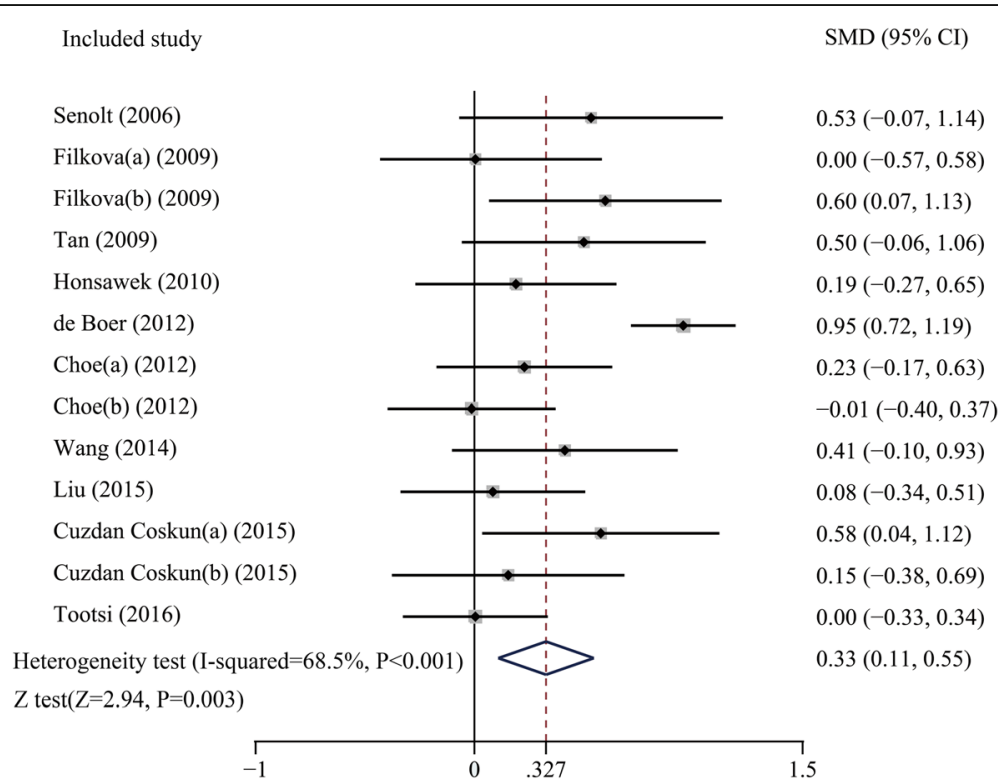

Fig. 2 Forest plot for the clinical significance of adiponectin expression level in osteoarthritis patients compared with healthy controls. SMD, standardized mean difference; Cl. confidence interval 
Table 2 Subgroup analyses stratified by various factors

\begin{tabular}{|c|c|c|c|c|c|c|c|c|}
\hline Factors & Subgroups & Studies (n) & Patients(n) (Case/Control) & $I^{2}(\%)$ & Heterogeneity $(p)$ & SMD & $95 \% \mathrm{Cl}$ & $Z$ test $(p)$ \\
\hline \multirow[t]{2}{*}{ Ethnicity } & Caucasians & 7 & $398 / 315$ & 77.7 & $<0.001$ & 0.42 & $0.06-0.71$ & 0.021 \\
\hline & Asians & 6 & $356 / 186$ & 0.0 & 0.661 & 0.19 & $0.01-0.18$ & 0.037 \\
\hline \multirow[t]{3}{*}{ Joint involvement } & Knee & 8 & $499 / 299$ & 65.7 & 0.005 & 0.44 & $0.17-0.72$ & 0.002 \\
\hline & Hand & 4 & $185 / 132$ & 20.1 & 0.289 & 0.18 & $-0.07-0.44$ & 0.161 \\
\hline & Knee and Hip & 1 & $70 / 70$ & - & - & 0.00 & $-0.33-0.34$ & 0.980 \\
\hline \multirow[t]{2}{*}{ Stage } & early OA & 2 & $77 / 66$ & 0.0 & 0.533 & 0.00 & $-0.57-0.58$ & 0.356 \\
\hline & Middle \& late OA & 11 & $677 / 435$ & 71.8 & $<0.001$ & 0.23 & $-0.17-0.63$ & 0.004 \\
\hline
\end{tabular}

OA on the basis of its negative correlation with disease progression [30, 31], and all of patients included in above studies was with middle and late OA. Interestingly, however, our pooled analyses also suggest that adiponectin was significantly up-regulated in middle and late stage of OA compared with healthy control. The expression of adiponectin might therefore be a pivotal biomarker that could help to the diagnosis of the progression of OA. Moreover, a study reported by StuM Otero et al. held the opinion that the increased levels of adiponectin in patients with arthritis suggested a compensatory mechanism under catabolic or anabolic imbalance [38]. Meanwhile, Aspden et al. in their study suggested that osteoarthritis is a systemic disorder in which promote lipid metabolism with its progression [39]. However, the circulating adiponectin level is natively associated with level of lipid metabolism [40]. Thus, we hypothesized that when the disease progressed, the adiponectin levels may be down-regulated in

Table 3 Meta-regression of single variable and multivariable

\begin{tabular}{|c|c|c|c|c|c|}
\hline Variables & Number of comparisons & $\beta$ & $95 \% \mathrm{Cl}$ & $P$ & $R^{2}$ \\
\hline \multicolumn{6}{|l|}{ Single variable } \\
\hline Publication Year & 13 & -0.03 & $-0.11-0.40$ & 0.40 & 0.68 \\
\hline Sample size & 13 & 0.0002 & $0.004-0.03$ & 0.03 & 0.53 \\
\hline Country(vs Czech Republic) & 13 & & & & 0.99 \\
\hline China & 3 & 0.99 & $-0.45-0.64$ & 0.67 & \\
\hline Thailand & 1 & -0.28 & $-0.83-0.26$ & 0.25 & \\
\hline Netherlands & 1 & -0.18 & $-0.68-0.31$ & 0.40 & \\
\hline Korea & 2 & 0.66 & $0.19-1.13$ & 0.01 & \\
\hline Turkey & 2 & -0.099 & $-0.78-0.58$ & 0.73 & \\
\hline Estonia & 1 & 0.072 & $-0.52-0.66$ & 0.77 & \\
\hline Location(vs knee) & 13 & & & & 0.16 \\
\hline Hand & 2 & 0.21 & $-0.20-0.73$ & 0.24 & \\
\hline Knee and Hip & 1 & 0.34 & $-0.95-0.57$ & 0.60 & \\
\hline Stage(secondary OA/primary OA) & 13 & 0.22 & $-0.39-0.85$ & 0.43 & 0.69 \\
\hline \multicolumn{6}{|l|}{ Multivariable } \\
\hline Mean of Age(years) & & & & & 0.02 \\
\hline $\mathrm{OA}$ & 13 & 0.02 & $-0.02-0.06$ & 0.28 & \\
\hline Control & 13 & -0.01 & $-0.04-0.01$ & 0.40 & \\
\hline Gender(female\%) & & & & & -0.09 \\
\hline $\mathrm{OA}$ & 13 & -0.002 & $-0.02-0.02$ & 0.98 & \\
\hline Control & 13 & 0.05 & $-0.01-0.02$ & 0.52 & \\
\hline Mean of BMI & & & & & 0.06 \\
\hline $\mathrm{OA}$ & 13 & -0.04 & $-0.16-0.08$ & 0.43 & \\
\hline Control & 13 & 0.10 & $-0.09-0.30$ & 0.26 & \\
\hline
\end{tabular}

A value of $P<0.05$ was considered statistically significant 


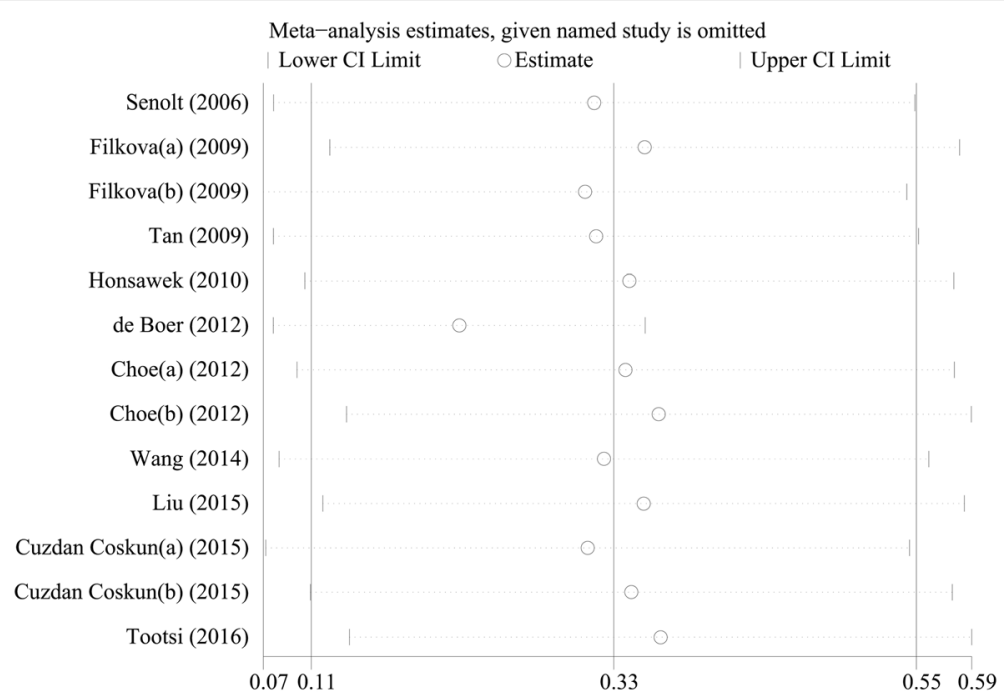

Fig. 3 Sensitivity analysis was performed through omitting individual studies one by one to evaluate whether the overall results could have been significantly influenced by one single study

response to the elevating level of lipid metabolism. It would provide a new therapy target for OA if our hypothesis be confirmed in the future.

The results of our subgroup analysis also revealed that this association was stronger in the patients with knee OA but might not with hand OA or knee and hip OA. Bias might be present for the knee and hip OA group as there was only one study in the literature. Therefore, the clinical applicability of our conclusion should be taken into consideration with other major diagnostic strategies in hand or hip OA patient. As for knee joint, one of weight-bearing joint most influenced by obesity, was confirmed to be more susceptibility through the action of inflammatory adipokines than hip joint [41]. This finding was consistent with our result. However, Arita et al. [15] discovered the adiponectin in obese subjects were significantly lower than that in non-obese one, although adiponectin was secreted only from adipose tissue. Which suggested a more complex relationship among obesity, adiponectin and OA and might further approve of our hypothesis that adiponectin increase compensatorily in the OA patient. Furthermore, the ethnicity-stratified analysis showed that the adiponectin expression level was higher in both Caucasian and Asian OA subjects than in the controls, which indicated ethnicity differences may not substantially affect the outcomes. In addition, we cannot get sufficient information about gender difference in this issue owing to result of meta-regression analyses, although

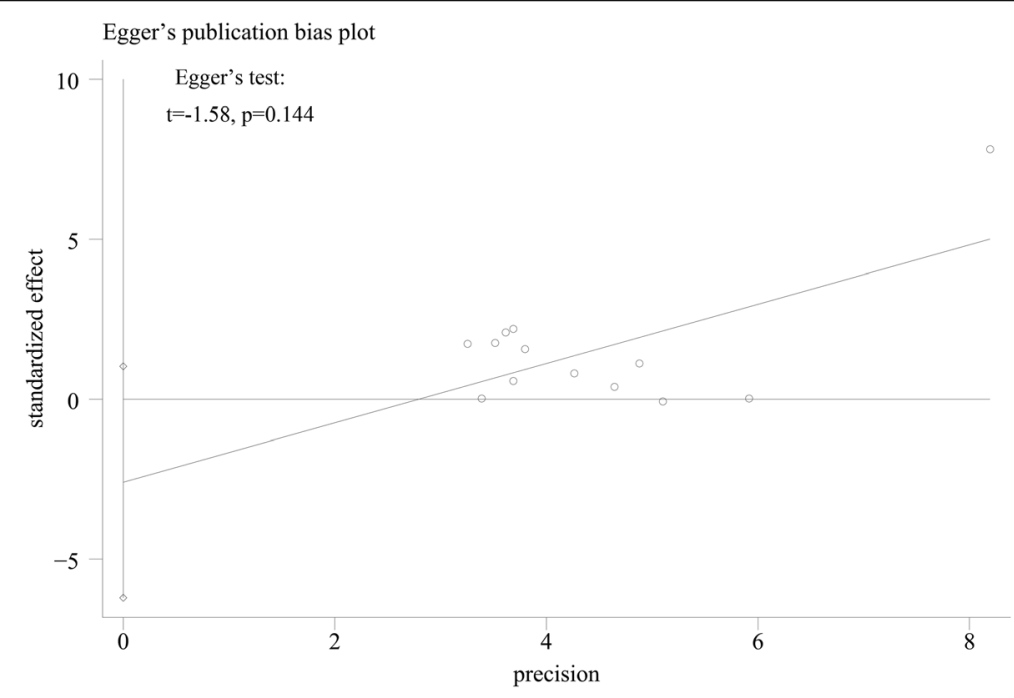

Fig. 4 Egger's plot was performed to evaluate the publication bias of included studies 
lots of researchers such as Perruccio et al. [42] have found that adiponectin level was higher in female than male, suggesting the adiponectin might be a sensitive predictor in women with OA.

However, it is worth mentioning that the significant heterogeneity was detected when we combined the SMD. Because the varied characteristics of all included studies might have influenced the heterogeneity such as sample size, study designs, patient characteristics. Therefore, we used a random-effect model to synthesize the data on the basis of large population. The subgroup study and metaregression analyses were performed to detected the potential sources of heterogeneity. In the result, we found that the sample size and nationality of patients might explain a part of heterogeneity, which suggested some high quality studies consisting of enough sample and varies of countries were needed to this issue in the future. Furthermore, the result of sensitive analyses and egger test were also supporting the stability of our conclusion.

Excepting heterogeneity, the current meta-analysis has several limitations. First, to improve the level of evidence, we restricted the inclusion criteria of included studies which must contained control group so that several cross-sectional studies were excluded. The data were therefore inadequate to estimate the potential relationship between the severity of the OA and the adiponectin levels. Therefore, based on our study, a further meta-analysis that will include all of the related observational studies will be necessary to evaluate the specificity of the association between adiponectin and the severity of OA. Second, in the ethnicity-stratified analysis, we did not have any information regarding mixed populations or black people, so the conclusion may not represent the worldwide distribution of ethnicities. Third, several unpublished papers and meeting abstracts were not taken into account because the data required for the inclusion and exclusion criteria was unavailable, which may cause a potential selection bias. In spite of these limitations, our study is the first meta-analysis to investigate the correlation between the adiponectin expression level and OA prevalence.

In conclusion, our meta-analysis revealed that the adiponectin expression level was up-regulated in later stage of OA especially in knee OA patients. Most importantly, these results may therefore provide a potential reliable tool for synergistically diagnosing $\mathrm{OA}$, demonstrating its pivotal clinical significance and might be a potential target for the OA therapy. Further research with standardized, unbiased methods and larger sample sizes are required for deeper analysis.

\section{Acknowledgements}

We would like to acknowledge the reviewers for providing helpful comments on this paper.

\section{Funding}

This study was supported by National Natural Science Foundation of China (NO.81501869, NO.81601983, NO. 41506091), Medical and health science and technology project of Zhejiang Province (2016YKA139), and Zhejiang provincial Public welfare project(2017C33035).

\section{Availability of data and materials}

The datasets used and analyzed during the current study are available from the corresponding author on request.

\section{Authors' contributions}

QT and HL designed the study. PS, ZCH and HX performed statistical analysis, and drafted the manuscript. QT, ZCH and LYS collected data and assisted with statistical analysis and manuscript drafting. All authors read and approved the final manuscript.

\section{Ethics approval and consent to participate}

This article does not contain any studies with human participants or animals performed by any of the authors.

\section{Consent for publication}

Not applicable.

\section{Competing interests}

The authors declare that they have no competing interests.

\section{Publisher's Note}

Springer Nature remains neutral with regard to jurisdictional claims in published maps and institutional affiliations.

\section{Author details}

${ }^{1}$ Department of Orthopaedic Surgery, The Second Affiliated Hospital and Yuying Children's Hospital of Wenzhou Medical University, 109, Xueyuanxi road, Wenzhou 325027, China. 'Department of Rehabilitation, The Second Affiliated Hospital and Yuying Children's Hospital of Wenzhou Medical University, 109, Xueyuanxi road, Wenzhou 325027, China.

Received: 10 September 2017 Accepted: 31 July 2018

Published online: 16 August 2018

\section{References}

1. Garstang SV, Stitik TP. Osteoarthritis: epidemiology, risk factors, and pathophysiology. Am J Phys Med Rehabil. 2006;85:S2-11. quiz S12-14

2. Glyn-Jones S, Palmer AJ, Agricola R, Price AJ, Vincent TL, Weinans H, Carr AJ. Osteoarthritis. Lancet. 2015:386:376-87.

3. Guillemin F, Rat AC, Mazieres B, Pouchot J, Fautrel B, Euller-Ziegler L, Fardellone P, Morvan J, Roux CH, Verrouil E, et al. Prevalence of symptomatic hip and knee osteoarthritis: a two-phase population-based survey. Osteoarthr Cartil. 2011;19:1314-22.

4. Goldring SR, Goldring MB. Bone and cartilage in osteoarthritis: is what's best for one good or bad for the other? Arthritis Res Ther. 2010:12:143.

5. Hiligsmann M, Cooper C, Arden N, Boers M, Branco JC, Luisa Brandi M, Bruyere O, Guillemin F, Hochberg MC, Hunter DJ, et al. Health economics in the field of osteoarthritis: an expert's consensus paper from the European Society for Clinical and Economic Aspects of osteoporosis and osteoarthritis (ESCEO). Semin Arthritis Rheum. 2013;43:303-13.

6. Gabay O. Osteoarthritis: new perspectives. J Spine. 2012;1:e101.

7. Wluka AE, Lombard CB, Cicuttini FM. Tackling obesity in knee osteoarthritis. Nat Rev Rheumatol. 2013;9:225-35

8. Sowers MR, Karvonen-Gutierrez CA. The evolving role of obesity in knee osteoarthritis. Curr Opin Rheumatol. 2010;22:533-7.

9. Gabay O, Hall DJ, Berenbaum F, Henrotin Y, Sanchez C. Osteoarthritis and obesity: experimental models. Joint Bone Spine. 2008;75:675-9.

10. Ibrahim SM, Hamdy MS, Amer N. Plasma and synovial fluid adipocytokines in patients with rheumatoid arthritis and osteoarthritis. Egypt J Immunol. 2008;15:159-70.

11. Lago R, Gomez R, Otero M, Lago F, Gallego R, Dieguez C, Gomez-Reino Ju, Gualillo O. A new player in cartilage homeostasis: adiponectin induces nitric oxide synthase type II and pro-inflammatory cytokines in chondrocytes. Osteoarthr Cartil. 2008;16:1101-9. 
12. Gross JB, Guillaume C, Gegout-Pottie P, Mainard D, Presle N. Synovial fluid levels of adipokines in osteoarthritis: association with local factors of inflammation and cartilage maintenance. Biomed Mater Eng. 2014;24:17-25.

13. Poonpet T, Honsawek S. Adipokines: biomarkers for osteoarthritis? World J Orthop. 2014;5:319-27.

14. Cuzdan Coskun N, Ay S, Evcik FD, Oztuna D. Adiponectin: is it a biomarker for assessing the disease severity in knee osteoarthritis patients? Int J Rheum Dis. 2017;20:1942-9.

15. Arita Y, Kihara S, Ouchi N, Takahashi M, Maeda K, Miyagawa J, Hotta K, Shimomura I, Nakamura T, Miyaoka K, et al. Paradoxical decrease of an adipose-specific protein, adiponectin, in obesity 1999. Biochem Biophys Res Commun. 2012;425:560-4.

16. Okamoto Y, Kihara S, Ouchi N, Nishida M, Arita Y, Kumada M, Ohashi K, Sakai N, Shimomura I, Kobayashi $\mathrm{H}$, et al. Adiponectin reduces atherosclerosis in apolipoprotein E-deficient mice. Circulation. 2002:106:2767-70.

17. Pischon T, Girman CJ, Hotamisligil GS, Rifai N, Hu FB, Rimm EB. Plasma adiponectin levels and risk of myocardial infarction in men. JAMA. 2004;291:1730-7.

18. Yamauchi T, Kamon J, Waki H, Terauchi Y, Kubota N, Hara K, Mori Y, Ide T, Murakami K, Tsuboyama-Kasaoka N, et al. The fat-derived hormone adiponectin reverses insulin resistance associated with both lipoatrophy and obesity. Nat Med. 2001;7:941-6.

19. Fantuzzi G. Adiponectin and inflammation: consensus and controversy. J Allergy Clin Immunol. 2008;121:326-30.

20. Tan W, Wang F, Zhang M, Guo D, Zhang Q, He S. High adiponectin and adiponectin receptor 1 expression in synovial fluids and synovial tissues of patients with rheumatoid arthritis. Semin Arthritis Rheum. 2009;38:420-7.

21. Kang EH, Lee YJ, Kim TK, Chang CB, Chung JH, Shin K, Lee EY, Lee EB, Song YW. Adiponectin is a potential catabolic mediator in osteoarthritis cartilage. Arthritis Res Ther. 2010;12:R231

22. Koskinen A, Juslin S, Nieminen R, Moilanen T, Vuolteenaho K, Moilanen E. Adiponectin associates with markers of cartilage degradation in osteoarthritis and induces production of proinflammatory and catabolic factors through mitogen-activated protein kinase pathways. Arthritis Res Ther. 2011;13:R184

23. Burrage PS, Mix KS, Brinckerhoff CE. Matrix metalloproteinases: role in arthritis. Front Biosci. 2006;11:529-43.

24. Liu X. Correlation between the incidence of osteoarthritis and the expression of adiponectin in patients with knee osteoarthritis. Chin J Ethnomed Ethnopharm. 2015;2:44-5.

25. Wang XY, Xue Q. Correlation between adiponectin in synovial fluid and knee osteoarthritis severity. Chin J Bone Joint Surg. 2014;7:96-100.

26. Senolt L, Pavelka K, Housa D, Haluzik M. Increased adiponectin is negatively linked to the local inflammatory process in patients with rheumatoid arthritis. Cytokine. 2006;35:247-52.

27. Filkova M, Liskova M, Hulejova H, Haluzik M, Gatterova J, Pavelkova A, Pavelka K, Gay S, Muller-Ladner U, Senolt L. Increased serum adiponectin levels in female patients with erosive compared with non-erosive osteoarthritis. Ann Rheum Dis. 2009;68:295-6.

28. de Boer TN, van Spil WE, Huisman AM, Polak AA, Bijlsma JW, Lafeber FP, Mastbergen SC. Serum adipokines in osteoarthritis; comparison with controls and relationship with local parameters of synovial inflammation and cartilage damage. Osteoarthr Cartil. 2012;20:846-53.

29. Tootsi K, Kals J, Zilmer M, Paapstel K, Martson A. Severity of osteoarthritis is associated with increased arterial stiffness. Int J Rheumatol. 2016;2016:6402963.

30. Honsawek S, Chayanupatkul M. Correlation of plasma and synovial fluid adiponectin with knee osteoarthritis severity. Arch Med Res. 2010;41:593-8.

31. Choe JY, Bae J, Jung HY, Park SH, Lee HJ, Kim SK. Serum resistin level is associated with radiographic changes in hand osteoarthritis: cross-sectional study. Joint Bone Spine. 2012;79:160-5.

32. Yusuf E, loan-Facsinay A, Bijsterbosch J, Klein-Wieringa I, Kwekkeboom J, Slagboom PE, Huizinga TW, Kloppenburg M. Association between leptin, adiponectin and resistin and long-term progression of hand osteoarthritis. Ann Rheum Dis. 2011;70:1282-4.

33. Zheng S, Xu J, Xu S, Zhang M, Huang S, He F, Yang X, Xiao H, Zhang H, Ding C. Association between circulating adipokines, radiographic changes, and knee cartilage volume in patients with knee osteoarthritis. Scand J Rheumatol. 2016;45:224-9.

34. Panic N, Leoncini $E$, de Belvis $G$, Ricciardi W, Boccia S. Evaluation of the endorsement of the preferred reporting items for systematic reviews and meta-analysis (PRISMA) statement on the quality of published systematic review and meta-analyses. PLoS One. 2013;8:e83138.
35. Altman R, Asch E, Bloch D, Bole G, Borenstein D, Brandt K, Christy W, Cooke TD, Greenwald R, Hochberg M, et al. Development of criteria for the classification and reporting of osteoarthritis. Classification of osteoarthritis of the knee. Diagnostic and therapeutic criteria Committee of the American Rheumatism Association. Arthritis Rheum. 1986;29:1039-49.

36. Stang A. Critical evaluation of the Newcastle-Ottawa scale for the assessment of the quality of nonrandomized studies in meta-analyses. Eur J Epidemiol. 2010;25:603-5.

37. Francin PJ, Abot A, Guillaume C, Moulin D, Bianchi A, Gegout-Pottie P, Jouzeau JY, Mainard D, Presle N. Association between adiponectin and cartilage degradation in human osteoarthritis. Osteoarthr Cartil. 2014:22:519-26.

38. Otero M, Lago R, Gomez R, Lago F, Dieguez C, Gomez-Reino JJ, Gualillo O. Changes in plasma levels of fat-derived hormones adiponectin, leptin, resistin and visfatin in patients with rheumatoid arthritis. Ann Rheum Dis. 2006;65:1198-201.

39. Richard M, Aspden BAAS, James D. Hutchison: osteoarthritis as a systemic disorder including stromal cell differentiation and lipid metabolism. Lancet. 2001;357:1118-20.

40. Staiger H, Tschritter O, Machann J, Thamer C, Fritsche A, Maerker E, Schick F, Haring HU, Stumvoll M. Relationship of serum adiponectin and leptin concentrations with body fat distribution in humans. Obes Res. 2003;11:368-72.

41. Mork PJ, Holtermann A, Nilsen TI. Effect of body mass index and physical exercise on risk of knee and hip osteoarthritis: Iongitudinal data from the Norwegian HUNT study. J Epidemiol Community Health. 2012;66:678-83.

42. Perruccio AV, Mahomed NN, Chandran V, Gandhi R. Plasma adipokine levels and their association with overall burden of painful joints among individuals with hip and knee osteoarthritis. J Rheumatol. 2014:41:334-7.
Ready to submit your research? Choose BMC and benefit from:
- fast, convenient online submission
- thorough peer review by experienced researchers in your field
- rapid publication on acceptance
- support for research data, including large and complex data types
- gold Open Access which fosters wider collaboration and increased citations
- maximum visibility for your research: over $100 \mathrm{M}$ website views per year
At $\mathrm{BMC}$, research is always in progress. 